\title{
Laboreal
}

Volume $3 \mathrm{~N}^{\circ} 2$ | 2007

Trabalho infantil

\section{INSAT : uma proposta metodológica para análise dos efeitos das condições de trabalho sobre a saúde}

INSAT : una propuesta metodológica para el análisis de los efectos de las condiciones de trabajo en la salud

INSAT : une proposition méthodologique pour l'analyse des effets des conditions de travail sur la santé

INSAT : a methodological proposal for the analysis of the effects work conditions have on health

Carla Barros-Duarte, Liliana Cunha e Marianne Lacomblez

\section{(2) OpenEdition}

\section{Journals}

Edição electrónica

URL: http://journals.openedition.org/laboreal/12554

DOI: $10.4000 /$ laboreal. 12554

ISSN: 1646-5237

Editora

Universidade do Porto

Refêrencia eletrónica

Carla Barros-Duarte, Liliana Cunha e Marianne Lacomblez, « INSAT : uma proposta metodológica para análise dos efeitos das condições de trabalho sobre a saúde », Laboreal [Online], Volume 3 №2 | 2007, posto online no dia 01 dezembro 2007, consultado o 10 outubro 2019. URL : http:// journals.openedition.org/laboreal/12554; DOI : 10.4000/laboreal.12554

Este documento foi criado de forma automática no dia 10 outubro 2019.

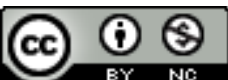

Laboreal está licenciado com uma Licença Creative Commons - Atribuição-NãoComercial 4.0 Internacional. 


\section{INSAT : uma proposta metodológica para análise dos efeitos das condições de trabalho sobre a saúde}

INSAT : una propuesta metodológica para el análisis de los efectos de las condiciones de trabajo en la salud

INSAT : une proposition méthodologique pour l'analyse des effets des conditions de travail sur la santé

INSAT : a methodological proposal for the analysis of the effects work conditions have on health

Carla Barros-Duarte, Liliana Cunha e Marianne Lacomblez

\section{NOTA DO EDITOR}

Manuscrito recebido em : Novembro/2007

Aceite após peritagem em : Dezembro/2007

\section{NOTA DO AUTOR}

O que fazer para ter acesso ao inquérito INSAT ?

Para conhecer o inquérito INSAT é solicitado que faça o pedido escrevendo uma mensagem electrónica às autoras do artigo (cujos endereços electrónicos estão indicados no início do artigo). No conteúdo desta mensagem devem figurar alguns dados pessoais (nomeadamente, o nome, a filiação profissional, ...), bem como as razões que explicam o facto de considerar útil ter acesso a este instrumento. Com base na justificação deste pedido, será ponderado o envio do inquérito.

Esta opção visa evitar possíveis utilizações abusivas do inquérito. Mas também visa facilitar o acesso das autoras às experiências desenvolvidas a partir do instrumento. 
Por estas razões, qualquer novo utilizador deverá fazer o seu pedido, sendo assim esperado que seja evitada a partilha do instrumento com utilizadores desconhecidos.

\section{Introdução}

1 As preocupações com o estudo da saúde e segurança no trabalho têm-se reflectido nos discursos e decisões políticas, o que, a nível nacional e internacional, se traduziu em mudanças significativas ao nível da legislação e, também, na criação de organismos orientados para um outro acompanhamento do seu cumprimento normativo.

Os dados mais recentes (Instituto de Informática e Estatística da Segurança Social, 2007) revelam que, paralelamente às doenças profissionais, como as doenças associadas ao aparelho respiratório e à surdez sonotraumática, as doenças músculo-esqueléticas e articulares, como as tendinites e as paralisias, têm ganho maior expressão ao nível dos dados estatísticos.

De facto, se os dados disponíveis ainda reflectem uma predominância das grandes patologias profissionais tradicionais (que não deixam de ser preocupantes), os outros problemas de saúde considerados até agora sem grande gravidade, começam a ganhar relevância e um outro estatuto, quando são discutidas as relações entre a saúde e o trabalho.

4 Na realidade, e como vem sublinhado no quarto e mais recente Relatório Europeu sobre as Condições de Trabalho (European Foundation for the Improvement of Living and Working Conditions, 2007), é fundamental analisar um conjunto de variáveis diferentes - as características de trabalho e as condições de emprego, a saúde e a segurança, a organização do trabalho, as oportunidades de desenvolvimento no trabalho e o balanço entre trabalho e não-trabalho -, de modo a ponderar melhor as consequências das condições de trabalho.

5 Torna-se, portanto, necessário adoptar uma outra perspectiva, já assumida por vários estudos científicos desenvolvidos na área da saúde e segurança no trabalho (entre outros, DaubasLetourneux \& Thébaud-Mony, 2002; Lacomblez, 2000; Derriennic, Touranchet \& Volkoff, 1996; Marquié, Paumès \& Volkoff, 1995; Teiger, 1989), de maneira a apreciar de forma mais completa os factores que interferem nas várias dimensões da saúde e abranger outros problemas e doenças provocadas pelas condições de trabalho, para além das doenças profissionais oficialmente reconhecidas (Gollac \& Volkoff, 2000).

6 Foi nesta perspectiva pensado e idealizado o Inquérito Saúde e Trabalho - INSAT - que se pretende configurar como uma proposta metodológica para a análise dos efeitos das condições de trabalho sobre a saúde.

\section{Problemática}

7 Se nos discursos científicos, e mesmo políticos, sobre a segurança e saúde no trabalho, tem vindo a emergir gradualmente uma maior sensibilização para o conhecimento da complexidade dos efeitos dos riscos profissionais, na realidade, as práticas desenvolvidas permanecem ainda aprisionadas a um conceito de saúde no trabalho equivalente ao de ausência de doença profissional, traduzindo-se, na maior parte das vezes, na prescrição de comportamentos ditos seguros e saudáveis. A mesma 
perspectiva é frequentemente associada à não integração das especificidades da vida de trabalho na abordagem global da saúde, o que acaba por "reforçar uma lógica, que tende a manter na periferia as preocupações relativas aos efeitos constrangedores do trabalho sobre o bem-estar e as acções susceptíveis de os diminuir" (Barros-Duarte \& Lacomblez, 2006, p. 1).

8 Uma abordagem com estas características, orientada por uma intervenção individualizada e de carácter normativo-prescritivo, é demasiado reducionista, limita os problemas de saúde aos que são susceptíveis de serem medicamente descritos (Davezies, 1994) e acaba por ignorar as verdadeiras consequências do trabalho na saúde.

9 As relações entre a saúde e o trabalho, na verdade, não são "nem unívocas nem instantâneas" (Gollac \& Volkoff, 2000, p. 23, tradução livre) - e desenvolvem-se a partir de um conjunto de fenómenos e estados que, embora não sejam considerados patológicos, constituem sinais de sofrimento (Marquié, 1999), muitas vezes, pouco apreendidos pelos diagnósticos tradicionais. Trata-se, nomeadamente, de um conjunto de problemas de saúde infra-patológicos, sem gravidade evidente: diminuição da audição, dores articulares, problemas de sono, sensação de fadiga, ansiedade ou consumo de tranquilizantes (Molinié \& Volkoff, 2002), susceptíveis de se concretizarem em problemas físicos ou psicológicos profundos, a que os investigadores e técnicos em saúde no trabalho se vêem, depois, confrontados nas suas práticas de prevenção.

10 Neste sentido, a integração de indicadores mais pertinentes, revelados por estudos de terreno, torna-se fundamental para avaliar as condições em que realmente se trabalha e em que medida favorecem mais ou menos a construção da saúde (Gollac

11 \& Volkoff, 2000). Esta não se reporta, então, a um estado mas a um processo dinâmico oscilando diariamente enquanto os trabalhadores vão gerindo as contradições entre a preservação da saúde e a garantia de produtividade, tentando contornar as possíveis fontes de penosidade ou de dificuldade (ThébaudMony, 2001 ; Volkoff, Molinié \& Jolivet, 2000).

12 Analisar as várias dimensões da saúde integrando a avaliação dos seus aspectos patológicos, mas também os outros problemas, que embora não sejam diagnosticados como patológicos influenciam o estado de bem-estar - os aspectos ditos infrapatológicos - quer do ponto de vista do especialista (médico), quer do ponto de vista do trabalhador (percepção do estado de saúde), torna-se, portanto, a única alternativa possível se quisermos evitar uma concepção de intervenção baseada na norma e no controlo (Cru, 2000) do comportamento do homem no trabalho.

13 Nesta perspectiva, o estudo da saúde no trabalho pode potencialmente suscitar uma reflexão a propósito da perspectiva assumida pelo trabalhador, traduzida nas suas declarações - nas percepções, nas queixas, e nos sentimentos acerca do seu trabalho e da sua saúde - e um alerta para uma nova abordagem nesta matéria.

14 É a este nível que encontramos um importante desafio na análise desta problemática : é fundamental assumir opções de carácter metodológico que permitam o reconhecimento plurifactorial dos problemas de saúde (Coutrot \& Wolff, 2005).

15 Vários inquéritos têm sido já concebidos e melhorados em interacção com as análises qualitativas e os conhecimentos epidemiológicos, procurando ilustrar a complexidade das condições de trabalho e das suas consequências no bem-estar. Três inquéritos que se enquadram nesta tradição são de seguida apresentados - o inquérito SUMER ; o 
inquérito EVREST ; e o inquérito SIT - por terem sido determinantes na construção do Inquérito Saúde e Trabalho - INSAT.

\section{Os pilares de construção do INSAT : os inquéritos SUMER, EVREST e SIT}

16 A construção do INSAT foi inspirada nos conhecimentos explorados por diferentes inquéritos, cujo contributo diferenciado aqui se reúne de forma sucinta. De referir que, a apresentação mais detalhada do inquérito SIT comparativamente com os outros instrumentos está directamente relacionada com a importância do contributo que acabou por ter na concepção do INSAT, o qual se encontra situado e melhor traduzido mais adiante.

\subsection{Inquérito SUMER - “ Surveillance Médicale des Risques Professionnels »}

O inquérito SUMER tem como objectivo estudar a penosidade do trabalho, através da recolha de informações sobre as condições de trabalho e a saúde dos trabalhadores. Trata-se de um inquérito francês, transversal, iniciado em 1987, sempre em colaboração com a inspecção médica do trabalho e os médicos do trabalho (Yilmaz, 2006). Posteriormente, ele foi reconduzido e ampliado aquando das suas aplicações em 1994/1995 e em 2002/2003 (Coutrot \& Wolff, 2005). Designadamente, à versão aplicada em 1994/1995 foi acrescentado um auto-questionário, cujo objectivo era permitir ao trabalhador "pronunciar-se sobre o vivido do seu trabalho e sobre o seu estado de saúde" (Yilmaz, 2006, p. 10, tradução livre), explorado pela primeira vez na versão de 2002/2003. Isto significa que a partir da organização desta nova versão o preenchimento do inquérito passou a ser realizado conjuntamente pelo médico do trabalho e pelo trabalhador.

18 É de salientar que as penosidades aqui consideradas são as que se mostram susceptíveis de contribuir para o desenvolvimento de patologias profissionais, afectando a longo prazo a saúde de forma grave e irreversível. Uma das principais dificuldades na sua declaração é pois devida ao tempo de latência (por vezes longo) destes efeitos sobre a saúde e ao facto de as exposições profissionais responsáveis nem sempre serem vividas como penosas.

19 Em termos metodológicos, a recolha de dados está organizada em 2 partes principais : (1) a primeira parte fornece informações sobre o trabalhador (sexo, idade, profissão exercida...) e sobre o sector em que trabalha; (2) a segunda parte diz respeito às exposições que sofreu o trabalhador - a duração da exposição semanal e a acumulação das penosidades -, a partir de uma lista de mais de 200 situações de trabalho, classificadas em 4 categorias: os constrangimentos organizacionais, os constrangimentos físicos, as exposições aos agentes biológicos e aos agentes químicos.

Relativamente ao auto-questionário introduzido na versão mais recente do inquérito, ele integra um questionário baseado na teoria de Karasek (1979), questões sobre a saúde percebida, a relação entre saúde e trabalho, a análise e avaliação dos resultados das medidas de redução do tempo de trabalho e ainda sobre a existência de problemas de carácter psicossocial. 
21 A originalidade deste inquérito reside no seu modo de administração : são os médicos do trabalho que retiram, aleatoriamente, os trabalhadores e administram o questionário (duração média de 30 minutos), no quadro da visita médica anual obrigatória para cada trabalhador. A resposta ao inquérito é centrada na actividade profissional realizada na semana anterior ao momento da aplicação (Coutrot \& Wolff, 2005).

\subsection{O Inquérito EVREST - “Évolutions et Relations en Santé au Travail »}

O inquérito EVREST tem como objectivo o conhecimento dos riscos do trabalho, da organização do trabalho, da saúde dos trabalhadores e das suas interacções. Trata-se de um inquérito francês, desenvolvido em 2001 pelos médicos da EADS [1], cujo objectivo era o de empreender acções capazes de melhorar a saúde no trabalho e, neste sentido, constituir uma rede de vigilância dos efeitos do trabalho - "rede EVREST" -, pilotada pelo CREAPT [ [2].

Os objectivos inerentes à concepção e aplicação do EVREST visam (Project EVREST, 2007) :

- Avaliar os níveis de risco e a saúde das populações no trabalho ;

- Acompanhar essas evoluções ;

- Avaliar as relações entre as exposições e a saúde ;

- Contribuir para a organização das prioridades a definir nas acções de prevenção ;

- Ajudar na avaliação das acções de prevenção.

A amostra do estudo congrega os trabalhadores nascidos em Outubro dos anos pares (o que corresponde a cerca de 1 trabalhador em cada 24) e ainda um conjunto de trabalhadores seleccionados aleatoriamente pelo médico do trabalho, independentemente do tipo de contrato que tenham com a empresa, à excepção dos trabalhadores temporários (Project EVREST, 2007).

O Inquérito é constituído por 2 partes principais : (1) na primeira parte, são solicitados dados de identificação do trabalhador e outras informações de carácter sóciodemográfico; (2) na segunda parte, são caracterizadas as condições de trabalho (as exposições profissionais) ; as formações ; o modo de vida e o estado de saúde.

o preenchimento do inquérito é partilhado pelo médico do trabalho (informações relativas ao estado de saúde), pelo trabalhador (exposições profissionais) e pelos serviços médicos (as informações de carácter geral). Os dados são recolhidos aquando da consulta anual de medicina do trabalho e conservados no dossier médico do trabalhador.

\subsection{O Inquérito SIT - "Saúde, Idade e Trabalho"}

27 O Inquérito SIT tem como objectivo principal compreender como e em que medida as condições de trabalho actuais e passadas influenciam, favorável ou desfavoravelmente, a qualidade do processo de envelhecimento e a saúde. Trata-se de um inquérito português, de carácter transversal, iniciado em 2001, e que teve a sua primeira aplicação no âmbito da participação na Campanha para a Melhoria das Condições de Trabalho na Indústria Têxtil e do Vestuário, promovida pelo IDICT [ ${ }^{3}$ ] (Barros-Duarte, 
Ramos, Cunha, \& Lacomblez, 2002). Dois outros sectores foram posteriormente integrados nos domínios de aplicação do inquérito SIT : o sector da cerâmica (Cunha, Costa, \& Lacomblez, 2003a, b) e, mais recentemente, o sector dos transportes (Cunha \& Gil Mata, 2005).

O Inquérito SIT foi concebido a partir dos contributos dos inquéritos ESTEV e VISAT (aplicados pela primeira vez nos anos de 1995 e 1996, respectivamente), tendo sido sobretudo o VISAT que, pela sua maior abrangência (valorização das dimensões psicológica e psicofisiológica das reacções aos efeitos das condições de trabalho), orientou o seu processo de construção.

O processo de construção deste instrumento, descrito em Barros-Duarte \& Lacomblez (2006), implicou um conjunto de etapas de adaptação a uma outra realidade linguística e social. Concretamente, e com referência àqueles que foram os seus inquéritos precursores (ESTEV e VISAT), certos itens foram incluídos, a formulação de algumas questões foi revista e foi reorganizada a exploração dos diferentes temas.

A estrutura do inquérito SIT reagrupa os itens em cinco questionários referentes aos seguintes domínios :

- "Características gerais e profissionais do trabalhador" : é constituído por questões gerais sobre o trabalhador e a profissão exercida ; por um conjunto de características relativas às condições de trabalho actuais e passadas (nomeadamente, a duração da exposição), com o objectivo de integrar uma análise de todo o percurso profissional do trabalhador ; por uma análise mais exaustiva do trabalho actual ; por uma avaliação das principais dificuldades sentidas pelo trabalhador na realização da sua actividade; por alguns índices relativos à qualidade do sono e do repouso ; e ainda por um conjunto de questões de (auto)apreciação do estado de saúde, que correspondem à aplicação da versão portuguesa do Perfil de Saúde de Nottingham $\left[{ }^{4}\right]$;

- "Questionário médico" : além da avaliação de alguns indicadores característicos do exame médico tradicional, inclui-se neste questionário um inventário de questões sobre patologias actuais e passadas, acidentes e doenças profissionais, assim como uma descrição dos medicamentos consumidos. Encontra-se ainda previsto um suplemento dirigido especificamente às mulheres, relativo a informações sobre a gravidez, a contracepção e a menopausa ;

- "Condições de vida fora do trabalho" : é constituído por um conjunto de questões relativas às características do trabalhador e à sua vida fora do tempo de trabalho, de modo a permitir uma compreensão mais ampla do que pode influenciar o seu percurso profissional e o modo de reagir a acontecimentos marcantes da vida ;

- “Testes psicotécnicos" : este questionário compila um conjunto de provas psicotécnicas clássicas que visam a recolha de dados relativos às capacidades cognitivas (memória, atenção, ...) ;

- "Exames complementares" : esta parte do inquérito integra alguns exames de medição de diferentes índices fisiológicos (capacidade pulmonar, visão e audição), com recurso a aparelhos médicos específicos para cada domínio.

31 A utilização do SIT em Portugal revestiu-se, portanto, de algumas particularidades relativamente aos inquéritos ESTEV e VISAT :

1. Um dos objectivos subjacentes à construção do SIT prende-se com uma preocupação de orientação para a intervenção e, nomeadamente, para o desenvolvimento de medidas preventivas e promotoras da saúde no trabalho. 
2. A sua estrutura contempla, como já foi referido, cinco questionários : em comparação com os três questionários presentes no Inquérito VISAT, o SIT proporcionou, deste modo, uma divisão temática mais especializada, que facilitou também a sua aplicação. Por outro lado, embora as temáticas presentes no inquérito SIT sejam muito semelhantes às dos inquéritos ESTEV e VISAT, foram introduzidos novos conteúdos, procurando responder à especificidade da organização da saúde no trabalho em Portugal e às características dos sectores em que foi aplicado (presente na selecção da amostra e na metodologia utilizada), assim como foram alteradas pontualmente algumas escalas de resposta.

3. Em termos metodológicos, foi assumida uma prática diferente da utilizada nos Inquéritos ESTEV e VISAT. A aplicação do SIT foi realizada em situação de entrevista individual, conjugando os contributos de uma equipa composta por três psicólogas do trabalho e médicos das empresas participantes. Procedeu-se, por isso, à construção de um guião de entrevista, baseado nos temas contemplados nos diferentes questionários, que acabou por facilitar o estabelecimento de uma relação de confiança com o trabalhador favorável à expressão da sua vivência no trabalho. A participação dos médicos garantiu um olhar atento na análise dos fenómenos associados à saúde : a sua posição privilegiada para a recolha sistemática de informações sobre a evolução da saúde foi, efectivamente, uma mais valia para este estudo.

4. Paralelamente, a complementaridade do estudo SIT com os contributos de uma análise qualitativa do trabalho e das condições de trabalho mostrou-se essencial para a melhor compreensão das análises estatísticas levadas a cabo com o SIT : as visitas e os contactos in loco com todas as empresas participantes permitiram um conhecimento mais aprofundado das características dos processos produtivos, das especificidades da organização do trabalho e das particularidades das condições de trabalho. Na verdade, este contacto directo com os contextos de trabalho revelou-se indispensável ao enquadramento e compreensão das verbalizações dos trabalhadores acerca das características do seu trabalho e das condições em que é realizado.

\subsection{Semelhanças e contributos diferenciados dos três inquéritos para o INSAT}


SUMER; a exploração do percurso profissional do trabalhador com referência às condições de trabalho actuais e passadas, presente nos inquéritos SUMER e SIT; a estrutura e organização das questões e das escalas de resposta relativamente ao estado de saúde, apresentada no inquérito EVREST; a integração de questões referentes às dificuldades sentidas no trabalho, representada no inquérito SIT; e a reflexão potenciada pela administração do inquérito sob a forma de entrevista, oferecida igualmente pelo inquérito SIT.

Por outro lado, de entre os aspectos comuns aos três inquéritos que foram incorporados no INSAT, podemos destacar : a escolha e selecção de questões já analisadas em estudos de terreno ; a referência às condições de vida fora do trabalho ; e finalmente (embora com expressão variável) a integração da perspectiva do trabalhador e a convocação da sua experiência subjectiva de percepção dos efeitos do trabalho na sua saúde.

\section{0 INSAT : Inquérito Saúde e Trabalho}

O Inquérito INSAT tem como objectivo estudar as consequências do trabalho e das condições de trabalho, actuais e passadas, ao nível da saúde e do bem-estar. Trata-se de um inquérito do tipo epidemiológico, que pretende neste momento caracterizar, através de uma amostra sectorial significativa, os principais riscos profissionais de alguns sectores de actividade e compreender a influência que os constrangimentos de trabalho têm na saúde do trabalhador.

Em termos dos objectivos específicos que lhe estão inerentes, podemos referir concretamente os seguintes :

- Elaborar uma caracterização das condições de trabalho associadas à actividade profissional em diferentes sectores de actividade ;

- Identificar os principais factores de risco e as interacções existentes entre eles, bem como a influência de certas variáveis relacionadas com o conteúdo de trabalho e a sua organização ;

- Oferecer aos investigadores um suporte para definir as prioridades de futuros estudos ;

- Definir medidas que possam promover uma melhoria das situações de trabalho e que, concomitantemente, minimizem os efeitos dessas situações sobre o risco de deterioração da saúde dos trabalhadores, no desempenho da sua actividade de trabalho ;

- Contribuir para que as empresas e os médicos do trabalho identifiquem os riscos a que se encontram expostos os trabalhadores e assim possam desenvolver uma carta de exposições a nocividades ou a situações de trabalho susceptíveis de serem prejudiciais à saúde ;

- Promover uma tomada de consciência individual e colectiva relativamente aos efeitos do trabalho e das condições de trabalho na saúde e bem-estar.

\subsection{Apresentação do instrumento}

O INSAT sustenta-se nos seguintes domínios : o trabalho e as condições de trabalho ; as dificuldades sentidas no trabalho, o estado de saúde e a saúde no trabalho. Estas temáticas foram incluídas na estrutura do INSAT, de modo a que as questões colocadas seguissem uma lógica coerente e integradora - do trabalho aos efeitos do trabalho na saúde - permitindo, a quem responde, uma tomada de consciência gradual e progressiva das consequências do trabalho na saúde e bem-estar. Condições e características do meu trabalho ; (III) Condições de vida fora do trabalho ; 
(IV) O que me custa mais no meu trabalho ; (V) Formação no meu trabalho ; (VI) O meu estado de saúde ; e (VII) Saúde no meu trabalho.

O inquérito tem início com algumas questões que permitem a recolha de informações, de carácter sócio-demográfico, sobre o trabalhador (sexo, idade, nível de escolaridade) e sobre o estabelecimento de emprego (sector de actividade, tipo de empresa, ...).

42 A primeira parte do inquérito - $\mathrm{O}$ meu trabalho - inclui um conjunto de questões relativas à situação de trabalho : corresponde à especificação e caracterização do tipo de actividade realizada, fazendo ainda referência ao tipo de vínculo laboral e ao horário de trabalho praticado.

43 Aquela que designamos como segunda parte do inquérito - Condições e características do meu trabalho - reúne as questões dedicadas à análise das exposições do trabalhador a determinadas condições, organizadas em três categorias: ambiente e constrangimentos físicos, nomeadamente ruído, vibrações, ambiente térmico, exposição a radiações, agentes biológicos e químicos, cargas, posturas, entre outros ; constrangimentos organizacionais e relacionais, nomeadamente tempos de trabalho; ritmos; autonomia e margens de iniciativa; relações de trabalho; contacto com o público; e características do trabalho, nomeadamente apreciações sobre o trabalho actual. Face a cada situação apresentada, o trabalhador deve responder se esteve ou não exposto ao constrangimento identificado. Se a sua resposta for afirmativa, deve assinalar se está exposto actualmente ou se a exposição ocorreu no passado e deve também identificar se a situação é ou foi desgastante, ou dito de outro modo, se a situação é ou foi causa possível de envelhecimento precoce.

44 A terceira parte corresponde à caracterização das - Condições de vida fora do trabalho -, sendo solicitado ao trabalhador informações sobre o número de pessoas a seu cargo, número de filhos e ocupação do tempo livre. A problemática da conciliação da vida de trabalho com a vida fora de trabalho é questionada, assim como o tempo despendido em tarefas domésticas e/ou de apoio familiar.

45 A quarta parte do Inquérito - 0 que me custa mais no meu trabalho - representa o vivido subjectivo do trabalhador face às condições a que se encontra exposto. Nesta parte, é pedido ao trabalhador que assinale o grau de penosidade que cada situação, a que se confronta no trabalho actual, representa para si. As questões que nos permitem caracterizar o tipo de formação realizada pelos trabalhadores e em que medida esta se relaciona ou não directamente com o trabalho enquadram-se na quinta parte do inquérito - Formação no meu trabalho.

46 Na sexta parte - 0 meu estado de saúde - são exploradas as questões relativas ao estado de saúde do trabalhador, sendo colocada a ênfase ao nível dos problemas que podem ter conduzido a determinadas limitações, mesmo que não se identifiquem com um quadro de patologia. Concretamente, os problemas referidos situam-se ao nível cardiorespiratório; neuro-psíquico; digestivo; muscular e articular. São ainda incluídas questões relativas ao estado de visão e de audição do trabalhador.

As questões mais específicas, relativas à saúde e trabalho, estão agrupadas na sétima e última parte do Inquérito - Saúde no meu trabalho - e em três domínios particulares : a identificação dos acidentes de trabalho e doenças profissionais que o trabalhador possa ter sofrido ou as situações que deflagraram numa doença profissional; informações sobre riscos profissionais, sendo incluídas neste campo questões que permitem obter informações relativas aos riscos profissionais e à protecção e prevenção contra eles; $\mathrm{e}$, 
no último domínio analisado, saúde e trabalho, são elencados vários problemas de saúde - físicos e psicossociais - que o trabalhador deve assinalar caso sinta ou tenha já sentido. Se a resposta for afirmativa é-lhe ainda solicitado um parecer sobre a relação desse problema ou queixa com o trabalho: isto é, se poderá ter sido causado ou agravado pelo trabalho. Neste caso, pretende-se que as questões colocadas favoreçam a análise da associação que o trabalhador estabelece, ou não, entre os seus problemas de saúde e as características e condições do seu trabalho actual ou passado.

De referir ainda que, o anonimato dos dados é assegurado por parte da equipa de investigação, uma vez que em momento algum é solicitado qualquer dado da identificação do trabalhador. Este é um procedimento deontológico de indiscutível relevo em estudos deste género, já que a sua negligência poderia comprometer a prossecução e validade do processo.

\subsection{Os desafios associados à construção do INSAT como questionário de administração directa}

Uma das particularidades do INSAT relativamente a outros inquéritos desenvolvidos com referência à problemática apresentada, em Portugal e no estrangeiro, consiste na forma como é preenchido. Efectivamente, o inquérito tal como é actualmente apresentado é o resultado de várias versões, que foram evoluindo até à forma actual com o objectivo de o tornar um instrumento de administração directa e centrado na perspectiva do trabalhador.

Esta opção é justificada pela intenção de atribuir ao trabalhador a possibilidade de se exprimir sem intermediário (contrariamente ao que acontece com os inquéritos anteriormente apresentados, em que esta administração é na sua essência mediada ora pelo médico, ora pelo psicólogo do trabalho) e, portanto, compreender de que forma os trabalhadores avaliam as características e condições do seu trabalho, o seu estado de saúde, e que tipo de relações estabelecem entre a sua saúde e o seu trabalho.

Se um dos principais contributos do INSAT se situa no facto de ter sido progressivamente concebido como auto-questionário, este constituiu, por outro lado, um dos seus maiores desafios: a utilização de outros inquéritos já desenvolvidos, nomeadamente o SIT, que fora aplicado especificamente à realidade portuguesa, havia permitido evidenciar a mais-valia de uma aplicação em contexto de entrevista (BarrosDuarte, Cunha, Ramos \& Lacomblez, 2001/2002), cujas respostas eram depois assinaladas no inquérito pelo psicólogo do trabalho e pelo médico do trabalho, a partir do discurso do trabalhador. Esta opção, além de se ter tornado identitária deste inquérito, favoreceu, por um lado, o desenvolvimento de uma reflexão por parte dos trabalhadores sobre a relação entre os factores associados à organização do trabalho e o sofrimento físico e psíquico vivenciado e, por outro lado, permitiu a emergência de um outro discurso sobre a saúde no trabalho.

Ora, apesar deste resultado que constitui em si uma vantagem oferecida pela metodologia entretanto adoptada na administração do SIT, a sua opção não deixou de comportar alguns custos que poderiam, eventualmente, comprometer a garantia de continuidade desta alternativa específica de aplicação. O tempo despendido com este formato foi um dos elementos mais invocados, uma vez que a aplicação do inquérito decorria durante o tempo de trabalho [5] e, no total, o tempo médio de aplicação do SIT era de cerca de 2 horas. 
O desafio que justificava a construção de um novo instrumento tornava-se então cada vez mais premente. No entanto, era esperado que a aplicação deste novo instrumento não só implicasse uma redução no tempo de preenchimento, como deveria manter a oportunidade de promover uma evolução na reflexão desenvolvida ao longo da resposta ao inquérito, como aconteceu com a metodologia do SIT.

54 Diferentes versões foram desenvolvidas e progressivamente optimizadas com referência a estes objectivos. Foi então concebido o INSAT como questionário de administração directa, destinado ao preenchimento pelo próprio trabalhador e a reflexão que outrora era suscitada pelo médico do trabalho ou pelo psicólogo do trabalho foi integrada na estrutura deste inquérito, através de uma evolução no tipo de questões colocadas (progressivamente vai sendo feito apelo a dimensões mais subjectivas, sendo pedido ao trabalhador, nomeadamente, para que enuncie, de entre as situações a que está exposto, as que se revelam mais penosas), de modo a permitir, paralelamente, uma tomada de consciência e uma evolução quer na reflexão desenvolvida, quer nas declarações assumidas ao longo do preenchimento do inquérito.

mente, aquela que corresponde à versão final do referido instrumento fo antecedida por versões preliminares, com o intuito de se verificar a adequação das perguntas e das respectivas escalas de resposta e o seu enquadramento face aos objectivos definidos. Concretamente, à medida que foram sendo testadas as suas diferentes versões com outros investigadores do mesmo domínio e médicos do trabalho, foram sendo introduzidas novas questões e instruções para o seu preenchimento, de modo a facilitar a compreensão do que era pedido.

No total, as várias versões do INSAT foram testadas com uma amostra de trabalhadores $(n=175)$ de diferentes sectores de actividade. A versão final do INSAT foi ainda submetida à metodologia da reflexão falada com 25 trabalhadores, que tiveram oportunidade de evidenciar e discutir as dificuldades na interpretação e resposta, isto é, relativamente ao conteúdo e formato em que eram colocadas as questões e as escalas de resposta.

5 No âmbito desta reflexão falada, que consistiu em solicitar aos trabalhadores comentários orais sobre a forma e o conteúdo do inquérito, acabaram por ser levantadas dificuldades que conduziram à reescrita de algumas questões, no sentido de as tornar mais claras para os respondentes, e à modificação de algumas escalas de resposta. A título exemplificativo, pode referir-se o facto de o inquérito inicialmente integrar escalas contínuas de resposta, que tiveram que ser alteradas e segmentadas em sete pontos diferentes (cada um dos quais correspondente a um determinado grau de resposta) : "concordo plenamente"; "concordo bastante"; "concordo parcialmente"; "nem concordo nem discordo"; "discordo parcialmente"; "discordo bastante"; e "discordo plenamente".

Para além deste exemplo, outros houve que reforçaram a necessidade de reformular o inquérito para o tornar mais coerente com o objectivo de se centrar na perspectiva do trabalhador e de tornar possíveis as declarações na primeira pessoa. Ora, se nas primeiras versões do INSAT, a quarta parte do inquérito era designada como Dificuldades sentidas no trabalho, as primeiras aplicações do inquérito e a reflexão falada tornaram evidente que o termo dificuldade era frequentemente interpretado de forma ambígua: encarado sobretudo como dificuldade de desempenho e não como dificuldade vivida na realização do trabalho, tal como era pretendido. Isto é tanto mais importante se considerarmos neste caso que, na óptica do trabalhador, quanto mais 
dificuldades fossem declaradas, tal significaria que mais inapto estaria para o trabalho. A alternativa encontrada foi ela própria sugerida pelos trabalhadores : esta parte do inquérito passou a ser identificada como $\mathrm{O}$ que me custa mais no meu trabalho. Diversos outros exemplos poderiam ser aqui retratados. Não obstante, todos eles remeteram para a necessidade de fazer no inquérito a transição do impessoal para a colocação das questões tal como se o trabalhador se estivesse a auto-interrogar. Fazê-lo conduz ainda a dotar este instrumento de uma função pedagógica, uma vez que se procura com esta mudança promover junto do trabalhador uma tomada de consciência gradual das consequências do seu trabalho na sua saúde. Por esta razão, o caminho até à concepção da versão final do INSAT foi sendo sempre orientado de forma a tornar o seu conteúdo o mais coerente possível com uma metodologia que se pretende de autoquestionário.

\subsection{Cuidados na análise e interpretação dos resultados}

INSAT, nomeadamente a integração de questões que se prendem com a evolução do trabalho e das opções de organização do trabalho, por outro lado, na apresentação do INSAT não poderiam deixar de ser assinaladas algumas preocupações a reter nos momentos de análise e interpretação dos resultados.

primeiro lugar, afirmamos que a aplicação do INSAT não pretende, nem pode, constituir uma alternativa exclusiva à análise do que se passa no terreno : da actividade tal como é realizada e das condições em que é desenvolvida. O INSAT, tal como foi concebido, recusa uma "tomada de assalto" ao terreno, assumindo em contrapartida o papel de um instrumento que coadjuva as análises da actividade real de trabalho.

Efectivamente, apesar das potencialidades estatísticas deste tipo de estudos, os autores costumam realçar o quanto as interpretações dos resultados deverão ser acompanhadas por uma reflexão prudente e cuidadosa na sua compreensão, tendo em consideração a complexidade de que se revestem as relações saúde e trabalho. Por isso, o recurso às pesquisas qualitativas assume uma importância crucial nas análises dos resultados estatísticos (Derriennic, Touranchet, \& Volkoff, 1996), nomeadamente os estudos de terreno, que recorrendo à análise da actividade de trabalho completam, enriquecem e confirmam os resultados obtidos (Volkoff, 1998). Aliás, e como já referido anteriormente, uma das preocupações presentes na formulação e concepção dos inquéritos, foi a de escolher e seleccionar questões que tivessem sido já analisadas e debatidas nos estudos de terreno, cujo método de análise assenta no conhecimento e na compreensão dos problemas reais em contexto real de trabalho (Volkoff, 1998).

Neste caso, afirma-se a importância de manter uma atitude reservada relativamente aos "scores" (Volkoff, 2002) e às possibilidades de generalizações, e a preocupação de comparar e integrar os resultados com os conhecimentos provenientes dos métodos qualitativos.

63 Efectivamente, a experiência com o estudo SIT havia já alertado para um possível confronto com resultados contraditórios entre o que emerge das análises da actividade e das entrevistas e o que se torna evidente com a utilização do método de inquérito.

É a este nível que se afirma a necessidade de adoptar uma abordagem multifacetada, capaz de ampliar as opções metodológicas da investigação. E isto é tanto mais importante se considerarmos que poder-se-ia correr o risco de produzir invisibilidade 
se uma análise mais aprofundada das interpretações de certas contradições nos resultados obtidos não fosse desenvolvida (Daubas-Letourneux, 2004).

A análise das relações menos visíveis entre a saúde e o trabalho, orientada por uma abordagem centrada no trabalhador, abre uma nova perspectiva de leitura dos seus efeitos. Isto significa que, a integração da perspectiva do trabalhador, traduzida nas percepções, nas queixas acerca da sua saúde, cuja expressão é potenciada pelo INSAT, associada a um parecer mais medicalizado, conduz a uma visão integradora e mais dinâmica das consequências do trabalho na saúde.

Neste sentido, o INSAT pode ser encarado como mediador do diálogo entre os actores envolvidos na prevenção, no quadro de uma intervenção que se pretende concertada. A abordagem que se torna visível no INSAT pode oferecer novas possibilidades de leitura da realidade e novas oportunidades de acção em contexto de trabalho, ao conduzir a investigação a tomar parte das declarações do trabalhador e nelas fundamentar as prioridades e o sentido dado à intervenção.

\section{BIBLIOGRAFIA}

Barros-Duarte \& Lacomblez (2006). Saúde no trabalho e discrição das relações sociais. Laboreal, 2, (2), 82-92. http://laboreal.up.pt/revista/artigo.php?id=37t45nSU547112278541446881

Barros-Duarte, C., Cunha, L., Ramos, S., \& Lacomblez, M. (2001/2002). Discursos e Métodos em Saúde no Trabalho. Cadernos de Consulta Psicológica, 17-18, 313-319.

Barros-Duarte, C., Ramos, S., Cunha, L. \& Lacomblez, M. (2002). Da organização do Trabalho à Saúde Ocupacional : análise das condições da actividade profissional na Indústria Têxtil e do Vestuário - a especificidade do trabalho feminino. Relatório apresentado no âmbito da Campanha para a melhoria das Condições de Trabalho no sector têxtil e do vestuário (em fase de publicação pelo ex-ISHST - ACT).

Coutrot, Th. \& Wolff, L. (2005). L'impact des conditions de travail sur la santé : une expérience méthodologique. Paris : Centre d'études de l'emploi (http://www.cee-recherche.fr).

Cru, D. (2000). Prévention et formes du dialogue social - Mise en perspective socio-historique. Revue de médicine du travail, XXVII, 2, 119-126.

Cunha, L. \& Gil Mata, R. (2005). Organização do Trabalho e Saúde Ocupacional no Sector dos Transportes : um contributo para a transformação das condições da actividade profissional dos motoristas. Relatório apresentado no âmbito do projecto de investigação para a melhoria das condições de trabalho no sector dos transportes (em fase de publicação pelo ex-IDICT - ISHST).

Cunha, L., Costa, A. \& Lacomblez, M. (2003a). Idade e Saúde na organização do trabalho : definir novas estratégias de prevenção dos riscos no sector da cerâmica. Revista Kéramica, 262, 50-58.

Cunha, L., Costa, A. \& Lacomblez, M. (2003b). Organização do Trabalho e Saúde Ocupacional no Sector da Cerâmica : Conhecer e Compreender a actividade para promover a Concepção de melhores condições de trabalho. Relatório apresentado no âmbito da Campanha para a melhoria das condições de trabalho no sector da cerâmica (em fase de publicação pelo ex-ISHST - ACT). 
Daubas-Letourneux, V. \& Thébaud-Mony, A. (2002). Organisation du travail et santé dans l'Union Européenne. Luxembourg: Office des Publications Officielles des Communautés Européennes.

Daubas-Letourneux, V. (2004). Accidents du travail : des accidents de parcours ? Regards croisés sur le (re)construction de la santé et sur le parcours professionnel de victimes d'accidents du travail. Les évolutions de la santé au cours de la vie professionnelle : altération, préservation, construction, séminaire Vieillissement et Travail, CRÉAPT-EPHE. Paris : Centre d'études de l'emploi (http://www.ceerecherche.fr).

Davezies, P. (1994). L'intervention sur la santé au travail : éléments de réflexion éthiques. Education Permanente, 121, 4, 131-143.

Derrienic, F., Touranchet, A. \& Volkoff, S. (1996). ESTEV, une méthode d'enquête. In Derrienic, Touranchet \& Volkoff (Eds.). Âge, travail, santé. Études sur les salariés âges de 37 à 52 ans. Enquête ESTEV 1990 (33-55). Paris : INSERM.

Derriennic, F., Touranchet, A. \& Volkoff, S. (1996). Âge, travail, santé. Paris : Les Éditions INSERM. European Foundation for the Improvement of Living and Working Conditions (2007). Fourth European Working Conditions Survey 2000. Luxembourg : Office for Official Publications of the European Communities.

Gollac, M. \& Volkoff, S. (2000). Les Conditions de Travail. Paris : Éditions La Découverte.

IIIP (2007). Dados estatísticos relativos às doenças profissionais : pensionistas com incapacidade permanente de 2001/2006. Instituto de Informática, Departamento de Gestão de Informação. Retirado em 22 de Outubro de 2007, de http://195.245.197.202/left.asp?02.21.03.07

Karasek, R. (1979). Job demands, job decision latitude, and mental strain : implications for job redesign. AdmSci., Quaterly, 24, 285308.

Lacomblez, M. (2000). Factores psicossociais associados aos riscos emergentes. Riscos emergentes da nova organização do trabalho. Lisboa : IDICT.

Marquié, J-C. (1999, Novembro). Quelques composantes psychiques et cognitives de la relation âge, travail, santé. Communication présentée au Colloque Santé, Travail, Vieillissement : Relations et Évolutions. CRÉAPT, Paris, France.

Marquié, J-C., Paumès, D., \& Volkoff, S. (1995). Le travail au fil de l'âge. Toulouse : Éditions Octares.

Molinié, A-F. \& Volkoff, S. (2002). La démographie du travail pour anticiper le vieillissement. Paris : ANACT.

Projet EVREST (2007). Évolution et Relations en Santé du Travail. European Aeronautic Defense Space Company. Retirado em Janeiro, 8, 2007 de https://194.254.117.103/EVREST/

Teiger, C. (1989). Le vieillissement différentiel par et dans le travail : un vieux problème dans un contexte récent. Le Travail Humain, 52, 1, 21-56.

Thébaud-Mony, A. (2001). L'impact de la précarité et de la flexibilité sur la santé des travailleurs. Numéro spécial - Bulletin d'Information du Bureau Technique Syndical Européen pour la Santé et la Sécurité, 15/16, 17-24.

Volkoff, S. (1998). Représentativité, significativité, causalité : l'ergonomie au contact des méthodes épidémiologiques. In M-F. Dessaigne \& I. Gaillard (Eds.), Des évolutions en ergonomie... (pp. 225-236). Toulouse : Éditions Octares. 
Volkoff, S. (2002). Des comptes à rendre : usages des analyses quantitatives en santé au travail pour l'ergonomie. Noisy-le-Grand : Centre d'Etudes de l'Emploi.

Volkoff, S., Molinié, A-F., \& Jolivet, A. (2000). Efficaces à tout âge ? Vieillissement démographique et activités de travail. Paris : Dossier du Centre d'Études de l'Emploi no. 16.

Yilmaz, E. (2006). Pénibilité du travail : évaluation statistique. Paris : Dossier du Centre d'Études de l'Emploi no. 55.

\section{NOTAS}

1. European Aeronautic Defense Space Company.

2. Centre de Recherches et d'Études sur l'Age et les Populations au Travail

3. Instituto de Desenvolvimento e Inspecção das Condições de Trabalho. Actualmente, este organismo assume a designação de Autoridade para as Condições de Trabalho (ACT).

4. Versão portuguesa, adaptada por investigadores da Faculdade de Economia da Universidade de Coimbra - Centro de Estudos e Investigação em Saúde - (1997), sob a responsabilidade do Prof. Doutor Pedro Lopes Ferreira.

5. À excepção do sector dos transportes, em que o SIT foi aplicado a uma amostra de motoristas e, tendo em conta que aplicá-lo durante o tempo de trabalho era incompatível com a actividade de trabalho (uma vez que quando estão ao serviço, os motoristas estão a conduzir), acabou por ser decidido que a sua administração teria que ocorrer fora do tempo de trabalho, embora pudesse ter lugar nas instalações das empresas implicadas.

\section{RESUMOS}

A problemática das relações saúde e trabalho não é nova, aliás, tem sido fonte de numerosos estudos e intervenções em vários países da União Europeia. Esta área de investigação é hoje objecto de um interesse particular devido, em parte, à obrigatoriedade no cumprimento da legislação e a uma maior sensibilização para o conhecimento dos efeitos dos riscos profissionais. A compreensão das consequências do trabalho na saúde e no bem-estar ganha vantagem no desenvolvimento de análises integradas e centradas na perspectiva do trabalhador sobre as condições em que trabalha. Inspirado em inquéritos já desenvolvidos e implementados, o instrumento que apresentamos - Inquérito Saúde e Trabalho (INSAT) - não só constitui um contributo nesta perspectiva, como pretende promover junto dos trabalhadores uma reflexão sobre os efeitos das condições de trabalho, tornando-os mais explícitos nas suas declarações.

La problemática de las relaciones salud y trabajo no es nueva, además, han sido fuente de numerosos estudios e intervenciones en varios países de la Unión Europea. Esta área de investigación es hoy objeto de un interés particular debido, en parte, a la obligatoriedad en el cumplimiento de la legislación y a una mayor sensibilización para el conocimiento de los efectos de los riesgos profesionales. La comprensión de las consecuencias del trabajo en la salud y en el bienestar gana ventaja en el desarrollo de análisis integradas y centradas en la perspectiva del trabajador sobre las condiciones en que trabaja. Inspirado en encuestas ya desarrolladas e implementadas, el instrumento que presentamos - Encuesta Salud y Trabajo (INSAT) - no sólo 
constituye una aportación en esta perspectiva, como pretende promover junto de los trabajadores una reflexión sobre los efectos de las condiciones de trabajo, haciéndolos más explícitos en sus declaraciones.

La problématique des relations entre santé et travail n'est pas nouvelle; d'ailleurs, elle a été source de nombreuses études et interventions dans divers pays de l'Union européenne. Ce domaine de recherche est aujourd'hui objet d'un intérêt particulier, dû entre autres à 1 'obligation de respect de la législation et, en conséquence, à une autre sensibilisation concernant l'intérêt d'une connaissance des effets des risques professionnels. La compréhension des conséquences du travail sur la santé et le bien-être a tout à gagner dans le développement $\mathrm{d}$ 'analyses intégrées, centrées sur la perspective du travailleur à propos des conditions dans lesquelles il travaille. Inspiré d'enquêtes antérieures, l'instrument que nous présentons Enquête Santé et Travail (INSAT) - se situe dans cette perspective. On prétend de plus l'utiliser afin de promouvoir chez les travailleurs une réflexion relative aux effets des conditions de travail, afin que ceux-ci soient davantage explicités dans les déclarations.

The problematic involving health and work relations is not new in itself. Actually, it has been a source of many studies and interventions in several EU countries. This research field is particularly interesting also due to the compulsory compliance with the legislation and to a greater awareness of the effects of professional risks. Understanding work consequences on workers' health and well being benefits from the development of an integrated analysis centred on workers' point of view and their working conditions. The tool we are presenting, the Health and Work Inquiry (INSAT), is based on other inquiries. Nevertheless, it gives a distinctive contribution to this integrated and worker-centred approach and also aims at promoting workers' reflections on the consequences of work conditions, making these effects more explicit in their statements.

\section{ÍNDICE}

Mots-clés: relations santé-travail, conditions de travail, questionnaire

Palabras claves: relaciones salud-trabajo, condiciones de trabajo, encuesta

Keywords: work-health relations, work conditions, questionnaire

Palavras-chave: relações saúde-trabalho, condições de trabalho, questionário

\section{AUTORES}

\section{CARLA BARROS-DUARTE}

Universidade Fernando Pessoa, Praça 9 de Abril, nº 349, 4249-004 Porto

cbarros@ufp.pt

\section{LILIANA CUNHA}

Universidade do Porto - Faculdade de Psicologia e de Ciências da Educação, Rua Dr. Manuel

Pereira da Silva, 4200-392 Porto

lcunha@fpce.up.pt 


\section{MARIANNE LACOMBLEZ}

Universidade do Porto - Faculdade de Psicologia e de Ciências da Educação, Rua Dr. Manuel Pereira da Silva, 4200-392 Porto

lacomb@fpce.up.pt 